\title{
ANÁLISE QUALI-QUANTITATIVA DA ARBORIZAÇÃO DAS ÁREAS PÚBLICAS DO BAIRRO CENTRO DE RESENDE, RJ
}

\author{
Mayara S. do Nascimento ${ }^{1}$, Eidilla da R. Rodrigues ${ }^{1}$, Carlos Alberto dos S. Souza ${ }^{1}$, \\ Maurício Jorge B. de Faria ${ }^{1}$, Jonas Pederassi ${ }^{2}$, Mauro Sergio C. S. Lima ${ }^{3}$
}

\section{RESUMO}

O estudo visou analisar aspectos fitossociológicos arbóreos de 7 áreas públicas do centro do município de Resende-RJ. Foram realizadas visitas as áreas entre os meses de março a maio de 2013 com um formulário para a coleta de dados e uma chave de identificação de espécies. Foram catalogados 171 indivíduos em 18 famílias e 43 espécies. Em relação à Diversidade de Shannon, a Praça do Centenário obteve o maior índice $\left(H^{\prime}=2,26\right)$ em relação às demais áreas avaliadas. O Parque do Aarão obteve o maior índice de Dominância de Simpson $(D=11,89)$. A Praça da Concórdia obteve o maior índice de Equitabilidade de Pielou $(\mathrm{J}=0,97)$. A Praça Marechal José Pessoa obteve o menor índice nos três aspectos supracitados $\left(H^{\prime}=0,79, D=1,65\right.$ e $J=0,57$ ). Quanto a fitossanidade das árvores, $43,27 \%$ dos espécimes foram considerados sadios e, apenas, $12,28 \%$ considerados ruins, demonstrando boa qualidade das árvores. A similaridade calculada por Jaccard variou de 0,07 a 0,23 , demonstrando uma dissimilaridade entre as áreas estudadas. Tal resultado pode ser devido à utilização de diferentes tipos de modelos paisagísticos pelas gestões municipais em diferentes épocas. As áreas avaliadas apresentaram uma elevada riqueza de espécies e boa qualidade, sendo necessário o conhecimento, respeito e preservação por parte da população.

Palavras-chave: Arborização urbana; Diversidades e equitabilidade; Similaridade; Fitossanidade.

\footnotetext{
Recebido em 05.06.2014 e aceito em 15.05.2015

${ }^{1}$ Centro Universitário de Barra Mansa (UBM), campus Barra Mansa. Rua Vereador Pinho de Carvalho, n 267. CEP 27.330550. Centro, Barra Mansa, RJ. E-mail: mayara_swim@yahoo.com.br

${ }_{2}^{2}$ UFRJ/MNRJ - Universidade Federal do Rio de Janeiro, Museu Nacional do Rio de Janeiro, Departamento de Vertebrados, Quinta da Boa Vista, CEP 20.940-040, São Cristóvão, Rio de Janeiro, RJ. Brasil.

${ }^{3}$ UFPI - Universidade Federal do Piauí, Campus Amilcar Ferreira Sobral, BR 343, km 3.5, Meladão, CEP 64.800-000, Floriano, PI. Brasil.
} 


\title{
QUALITATIVE-QUANTITATIVE ANALYSIS OF URBAN FORESTRY IN PUBLIC AREAS OF THE DOWNTOWN OF RESENDE-RJ
}

\begin{abstract}
This research aimed to analyze arboreal phytosociological aspects of 7 public areas at downtown of Resende - RJ. The areas were visited between the months of March to May 2013 with a form used for data collection and a species identification key. 171 individuals of 18 families and 44 species, were cataloged. The highest Diversity Index of Shannon was obtained at Centenário Square $\left(\mathrm{H}^{\prime}=2.26\right)$, compaired with the other evalueted areas. The Aarão Park had the highest Dominance Index of Simpson $(D=11.89)$. At the Concórdia Square was obtained the highest Equitability Index of Pielou $(\mathrm{J}=0.97)$. The Marechal José Pessoa Square had the lowest index in the three aspects mentioned above $\left(H^{\prime}=0.79\right.$, $\mathrm{D}=1.65$ and $\mathrm{J}=0.57$ ). About health of the trees , $43.27 \%$ of the specimens were considered healthy, and only $12.28 \%$ were considered bad, demonstrating good quality of the trees. The similarity calculated by Jaccard between the sampled areas ranged from 0.07 to 0.23 , demonstrating a dissimilarity between the studied areas. This result may be due to the different types of landscape models used in each municipal management in different periods. The evaluated areas showed a high species richness and a good quality, so is necessary the knowledge, respect and preservation by the population.
\end{abstract}

Keywords: Urban forestry; Diversity and equitability; Similarity; Health.

\section{INTRODUÇÃO}

O crescimento acelerado das cidades, resultante do acentuado aumento populacional, tem comprometido a qualidade de seus espaços livres e áreas verdes. Considerar a existência de benefícios econômicos e sociais das árvores nas cidades é apenas um processo lógico, uma vez que existem benefícios de ordens ecológica (clima e poluição), biológica e psicológica (respectivamente, saúde física e mental do ser humano) (LIRA et al., 2004). 
Desta forma, a arborização urbana representa o conjunto de terras públicas e privadas, com vegetação predominantemente arbórea que uma cidade apresenta, ou ainda, é um conjunto de vegetação arbórea natural ou cultivada de uma cidade em áreas particulares, praças, parques e vias públicas (SANCHONETE, 1994; SILVA JUNIOR; MÔNICO, 1994).

Segundo Dantas e Souza (2004), a arborização urbana no Brasil iniciou a pouco mais de 120 anos, sendo assim uma prática relativamente nova em comparação com os países europeus. Atualmente, em cidades onde ocorre o planejamento da arborização a preocupação é tornar o ambiente urbano diversificado quanto às espécies empregadas, tornando mais homogêneo e envolvente com a paisagem circundante (ROMANI et al., 2012).

Em contrapartida, as praças são locais representativos para distintas manifestações ao permitir a formação e agregações sociais (SILVA, 2009), e quando arborizadas são importantes espaços sócio-educativos, culturais e ambientais.

Os espaços arborizados ao ar livre amenizam o congestionamento domiciliar, oferecem espaços mais adequados e criativos às crianças, trazem saúde física e psicológica, aumentam a satisfação com seu local de moradia, colaboram para a redução de estresses cotidianos, além de outros benefícios (PAIVA, 2006).

Contudo, a arborização de um local sem planejamento pode acarretar em muitos conflitos com a estrutura urbana (MIRANDA; CARVALHO, 2009). Outro fator importante para um planejamento é a prioridade às plantas nativas, pois as espécies exóticas podem causar diversos danos ao ambiente como a perda da biodiversidade, modificações nos ciclos e características naturais dos ecossistemas atingidos, alteração fisionômica da paisagem natural e, algumas vezes consequências econômicas vultosas (ZILLER, 2001).

O inventário da arborização urbana tem como objetivo geral conhecer o patrimônio arbustivo e arbóreo de uma localidade. Tal estudo é fundamental para o planejamento e manejo da arborização, fornecendo informações sobre a necessidade de poda, tratamentos fitossanitários ou remoção e plantios, bem como para definir prioridades de intervenções ao ponto de se verificar os erros e acertos na arborização de uma cidade (MELO et al., 2007).

A cidade de Resende (às margens do Rio Paraíba do Sul) apresenta elevado crescimento sócio-econômico e está entre as cidades mais arborizadas do Estado, entretanto não se sabe qual é o estado atual das espécies plantadas em algumas áreas urbanas do centro de Resende, o que suscita alguns questionamentos: (1) Quais espécies foram utilizadas no paisagismo das áreas urbanas? (2) Qual é a condição fitossanitária das 
espécies vegetais encontradas? (3) Existe similaridade de espécies entre as áreas analisadas?

Neste contexto, o estudo visou analisar aspectos fitossociológicos arbóreos das áreas públicas do centro do município de Resende, como forma de gerar informações que subsidiem futuras ações de manejo e incentivem o uso das praças e dos parques como áreas para estudo.

\section{MATERIAIS E MÉTODOS}

O município de Resende (RJ) localiza-se no Sul Fluminense, mais precisamente na Região do Médio Vale do Paraíba, a $142 \mathrm{~km}$ do centro da cidade do Rio de Janeiro $\left(22^{\circ} 27^{\prime} \mathrm{S}\right.$ e $44^{\circ} 27^{\prime} O$ ), e possui uma área com cerca de $1.095 \mathrm{~km}^{2}$ (IBGE, 2010a) estando a 394,6 metros de altitude. O clima é considerado tropical de altitude, com temperatura média anual e precipitação média de $25^{\circ} \mathrm{C}$ e $1.500 \mathrm{~mm}$, respectivamente (PMR, 2013).

Foram inventariadas sete praças e um parque no centro da cidade de Resende (Figura 1) entre os meses de março a maio de 2013 para a caracterização das áreas públicas (Tabela 1).

Figura 1. Localização das áreas amostradas no centro da cidade de Resende-RJ

Figure 1. Location of areas sampled in the downtown of Resende-RJ

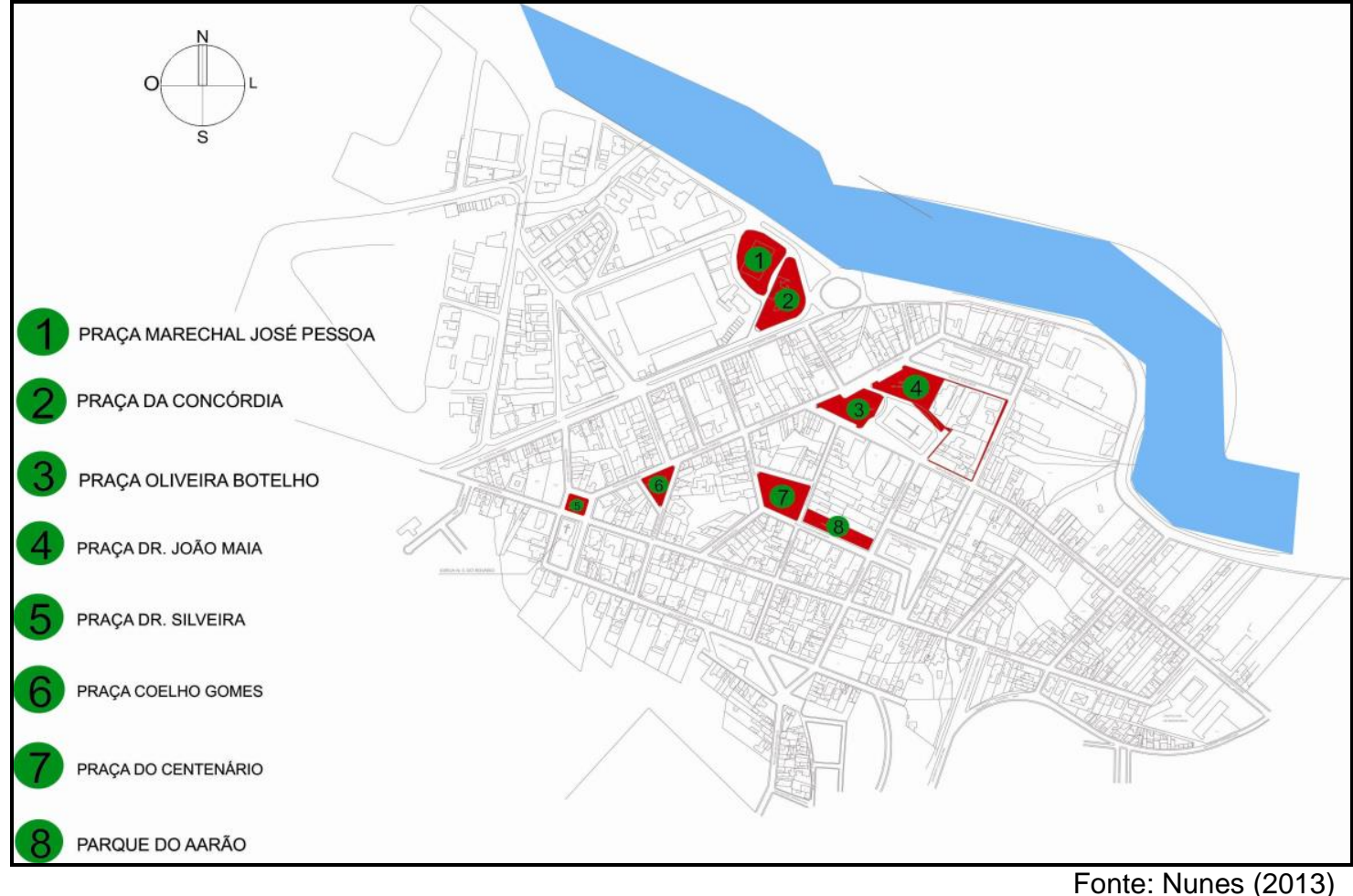


Tabela 1. Localização e dimensões das áreas amostradas no centro da cidade de Resende-RJ

Table 1. Location and dimensions of the areas sampled in the downtown of Resende-RJ

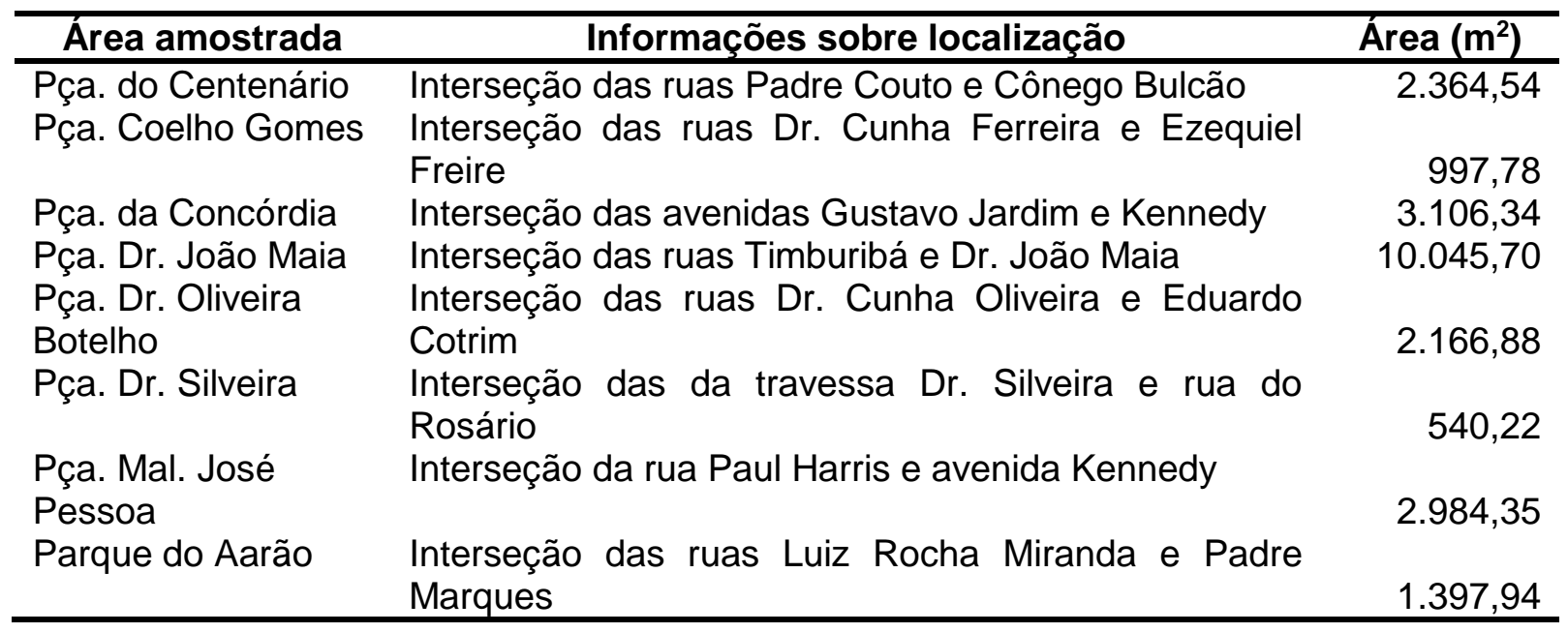

Antes de percorrer cada área, foi marcado um ponto de partida inicial representado pela interseção de ruas ou pontos de referências para facilitar o retorno à árvore-marco, caso fosse necessário.

A catalogação das árvores foi realizada conforme metodologia de cobertura adotada pelo IBGE (2010b), sendo que o critério de inclusão de indivíduos foi o DAP $\geq 10 \mathrm{~cm}$. Além disso, formulários específicos (modificado de MELO et al., 2007) foram preenchidos considerando variáveis quantitativas ou categóricas, como: aspectos estruturais [DAP $(\mathrm{cm})$ e altura $(m)$ ], aspectos biológicos (identificação taxonômica, presença/ausência de flores e frutos), ecológicos e de fitossanidade [presença/ausência de epífitas, liquens, briófitas, parasitas e doenças; sinais de podas (drástica/condução); sinais de danos às raízes, tronco e copa].

Todos os indivíduos aceitos no critério de inclusão foram analisados, fotografados e identificados taxonomicamente em campo (quando possível). As espécies não identificadas em campo foram coletadas e enviadas ao Laboratório Multidisciplinar do Centro Universitário de Barra Mansa para preparação e montagem de exsicatas e a identificação realizada com auxílio da obra bibliográfica de Lorenzi (2002a, b) ou de especialistas. Os binômios científicos foram corrigidos e atualizados por meio do Sistema de Classificação APG III (2009). Os nomes dos autores das espécies seguiram Brummitt e Powel (1992).

A frequência relativa foi calculada para as espécies amostradas entre as áreas de ocorrência e para todo o estudo. A diversidade entre as áreas foi determinada através do índice de Shannon $\left(H^{\prime}\right)$ e o índice de Simpson $\left(D_{(1 / D)}\right)$, enquanto a avaliação da equitabilidade foi aferida por Pielou (J) (MAGURRAN, 2004). Análise de agrupamento 
referente à similaridade de espécies entre as áreas amostradas foi realizada a partir do software Past $\AA$ (HAMMER et al., 2011).

\section{RESULTADOS E DISCUSSÃO}

\section{Composição da diversidade amostrada}

Foram amostrados 171 indivíduos de 18 famílias divididas em 43 espécies, sendo que cinco espécimes foram identificados somente até família e dois espécimes encontrados mortos foram considerados morfo-espécies (Tabela 2). As espécies de maior ocorrência do estudo foram Licania tomentosa (Benth) Fritsch seguida de Terminalia catappa L. e Livistona chinesis R. Brown ex Mart, onde juntas foram responsáveis por 35,0\% ( $\mathrm{N}=60)$ do total amostrado, sendo que as demais espécies não ultrapassou 10,0\%.

Tabela 2. Frequência das famílias e espécies total.

Table 2. Frequency of families and the total species.

\begin{tabular}{|c|c|c|c|}
\hline \multirow{2}{*}{ Táxons } & \multirow{2}{*}{ Origem } & \multicolumn{2}{|c|}{ Frequência relativa $(\%)$} \\
\hline & & $\mathbf{N}_{\mathbf{i}}$ & $\mathbf{N}_{\mathrm{F}}$ \\
\hline \multicolumn{4}{|l|}{ Anacardiaceae } \\
\hline Astronium graveolens Jacq. & $E_{n}$ & 0,58 & \multirow{3}{*}{1,75} \\
\hline Mangifera indica L. & $E_{e}$ & 0,58 & \\
\hline Spondias mombin L. & $E_{n}$ & 0,58 & \\
\hline \multicolumn{4}{|l|}{ Arecaceae } \\
\hline Dypsis lutescens (H.Wendl.) Beentje \& J. Dransf. & $E_{e}$ & 3,51 & \multirow{4}{*}{14,62} \\
\hline Livistona chinensis R. Brown ex Mart & $E_{e}$ & 7,60 & \\
\hline Roystonea oleracea (N. J. Jacquin) O.F. Cook & $E_{e}$ & 1,17 & \\
\hline Syagrus romanzoffiana (Cham.) Glassm & $E_{n}$ & 2,34 & \\
\hline \multicolumn{4}{|l|}{ Bignoniaceae } \\
\hline Handroanthus chrysotrichus (Mart. ex DC.) Mattos & $E_{n}$ & 1,17 & \multirow{7}{*}{15,79} \\
\hline Handroanthus heptaphylla (Vell.) Mattos & $E_{n}$ & 5,85 & \\
\hline Handroanthus impetiginosus (Mart. ex DC.) Mattos & $E_{n}$ & 2,92 & \\
\hline Handroanthus ochraceus (Cham.) Mattos & $E_{n}$ & 1,17 & \\
\hline Handroanthus vellosoi (Toledo) Mattos & $E_{n}$ & 2,92 & \\
\hline Tabebuia insignis (Miq.) Sandwith & $E_{n}$ & 0,58 & \\
\hline Tabebuia roseoalba (Rindl) Sandwith & $E_{n}$ & 1,17 & \\
\hline \multicolumn{4}{|l|}{ Casuarinaceae } \\
\hline Casuarina sp. & $E_{e}$ & 0,58 & 0,58 \\
\hline \multicolumn{4}{|l|}{ Chrysobalanaceae } \\
\hline Licania tomentosa (Benth) Fritsch & $E_{n}$ & 18,71 & 18,71 \\
\hline \multicolumn{4}{|l|}{ Combretaceae } \\
\hline Terminalia catappa L. & $E_{e}$ & 8,77 & 8,77 \\
\hline
\end{tabular}




\begin{tabular}{|c|c|c|c|}
\hline Fabaceae & & & \\
\hline Acacia sp. & - & 1,17 & \\
\hline Anadenanthera macrocarpa Benth. Brenan & $E_{n}$ & 1,75 & \\
\hline Bauhinia variegata $\mathrm{L}$. & $E_{e}$ & 0,58 & \\
\hline Caesalpinia echinata Lam. & $E_{n}$ & 1,17 & \\
\hline $\begin{array}{l}\text { Caesalpinia ferrea Mart. ex Tul. var. leiostachya } \\
\text { Benth. }\end{array}$ & $E_{n}$ & 0,58 & \\
\hline Caesalpinia peltophoroides Benth & $E_{n}$ & 2,92 & 15,20 \\
\hline Caesalpinia pulcherrima L. SW. & $E_{e}$ & 1,17 & \\
\hline Cassia fistula $\mathrm{L}$ & $\mathrm{E}_{\mathrm{e}}$ & 2,34 & \\
\hline Cassia grandis L.f. & $E_{n}$ & 0,58 & \\
\hline Leucaena leucocephala (Lam.) de Wit. & $E_{e}$ & 1,17 & \\
\hline Plathymenia foliolosa Benth. & $E_{n}$ & 1,17 & \\
\hline Senna spectabilis DC. H.S.Irwin \& Barneby & $E_{n}$ & 0,58 & \\
\hline Lauraceae & & & \\
\hline Persea americana Mill. & $E_{n}$ & 1,75 & 1,75 \\
\hline Lecythidaceae & & & \\
\hline Lecythis pisonis Camb. & $E_{n}$ & 2,34 & 2,34 \\
\hline Lythraceae & & & \\
\hline Lagerstroemia speciosa L. Pers. & $E_{e}$ & 0,58 & 0,58 \\
\hline Magnoliaceae & & & \\
\hline Michelia champaca L. & $\mathrm{E}_{\mathrm{e}}$ & 0,58 & 0,58 \\
\hline Moraceae & & & \\
\hline Ficus benjamina $\mathrm{L}$. & $\mathrm{E}_{\mathrm{e}}$ & 2,34 & \\
\hline Morus nigra L. & $\mathrm{E}_{\mathrm{e}}$ & 0,58 & 2,92 \\
\hline Myrtaceae & & & \\
\hline Eugenia brasiliensis Lam. & $E_{n}$ & 3,51 & \\
\hline Eugenia uniflora L. & $E_{n}$ & 0,58 & \\
\hline Syzygium cumini L. Skeels & $E_{e}$ & 1,17 & 5,85 \\
\hline Syzygium jambos L. Alston & $\mathrm{E}_{\mathrm{e}}$ & 0,58 & \\
\hline Nyctaginaceae & & & \\
\hline Bougainvillea glabra Choisy & $E_{n}$ & 0,58 & 0,58 \\
\hline Polygonaceae & & & \\
\hline Triplaris americana L. & $E_{n}$ & 1,17 & 1,17 \\
\hline Rhamnaceae & & & \\
\hline Hovenia dulcis Thunb. & $\mathrm{E}_{\mathrm{e}}$ & 2,92 & 2,92 \\
\hline Ruscaceae & & & \\
\hline Dracaena marginata Lam. & $\mathrm{E}_{\mathrm{e}}$ & 0,58 & 0,58 \\
\hline Sapindaceae & & & \\
\hline $\begin{array}{l}\text { Cupania oblongifolia Mart. } \\
\text { Sapindus saponaria L. }\end{array}$ & $\begin{array}{l}E_{n} \\
E_{n}\end{array}$ & $\begin{array}{l}0,58 \\
0,58\end{array}$ & 1,17 \\
\hline Morta & & 1,17 & 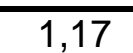 \\
\hline Sem identificação & & 2,92 & 2,92 \\
\hline Total & & 100,00 & 100,00 \\
\hline
\end{tabular}


Para Santamour Júnior (1990), a maior diversidade de espécies de árvores na paisagem urbana se faz necessária justamente para garantir o máximo de proteção contra pragas e doenças. Dessa forma, recomenda-se não exceder mais que 10,0\% da mesma espécie, $20,0 \%$ de algum gênero e $30,0 \%$ de uma família botânica. Portanto, somente a espécie $L$. tomentosa ultrapassa o recomendado.

Predomínio de $L$. tomentosa na arborização urbana de outras localidades também foi observado por Costa e Higuchi (1999) em Manaus-AM, Rossatto et al. (2008) em Assis-SP e Brianezi et al. (2013) em Viçosa-MG.

A grande frequência do L. tomentosa nas cidades está condicionada a sua copa frondosa e, por isso, tem sido largamente usada para fornecer sombra em arborização urbana por quase todo o Brasil, especialmente a partir do estado de São Paulo em direção ao Norte do país, mas o seu uso na arborização deve ser evitado em grande escala devido a uma doença causada por um fungo, que tem atacado a espécie frequentemente (FERREIRA et al., 2001).

Os resultados apontaram uma predominância de espécies nativas $(58,48 \% ; N=100)$ em relação às exóticas. As espécies exóticas com maior frequência foram $T$. catappa e $L$. chinesis (8,77 e 7,60\% respectivamente). Apesar do uso de espécies exóticas ser uma prática comum à grande maioria das cidades brasileiras no paisagismo de áreas públicas (parques, praças e jardins), os resultados obtidos entre as áreas amostradas na cidade de Resende não são muito preocupantes, pois o município encontra-se nas proximidades do Parque Nacional do Itatiaia (PARNA-Itatiaia). Espécies exóticas que se tornam invasoras podem se alastrar por diferentes áreas descontroladamente e trazer novas enfermidades ou pragas para espécies nativas.

Os valores obtidos pelo índice de Shannon apontaram que a Praça do Centenário apresentou maior diversidade $\left(H^{\prime}=2,26\right)$, enquanto a Praça Marechal José Pessoa o menor valor encontrado $\left(H^{\prime}=0,79\right)$. Esses resultados demonstraram que nem sempre a diversidade de árvores plantadas possui relação com o tamanho das áreas, já que a presença de edificações (prédios, igrejas, escolas e entre outras) em algumas das áreas estudadas resulta na redução do espaço público para plantio.

O índice de dominância de Simpson, que pondera a dominância das espécies, demonstrou que no Parque do Aarão houve maior dominância $\left[D_{(1 / D)}=11,89\right]$ em relação às outras áreas estudadas (Tabela 3). Possivelmente, os valores obtidos se devem a catalogação de 15 espécies, aonde nove espécies apresentaram apenas um exemplar, resultando em maior dominância de uma espécie e pouca diversidade. Este padrão também 
foi obtido nos estudos de Teixeira (1999), Lindenmaier e Santos (2008) e Lindenmaier e Souza (2014).

Excetuando a Praça Marechal José Pessoa, a equitabilidade de Pielou variou de $J=0,70$ a $J=0,97$ entre as outras áreas estudadas, demonstrando que todas as áreas tendem a uma distribuição uniforme (Tabela 3).

Tabela 3.Parâmetros ecológicos apresentados nas áreas estudadas.

Table 3. Ecological parameters presented in the studied areas.

\begin{tabular}{lcccccccc}
\hline \multirow{2}{*}{ Parâmetros Ecológicos } & \multicolumn{8}{c}{ Áreas amostradas } \\
\cline { 2 - 10 } & Sil & CoG & Con & MJP & Cen & JoM & OIB & PaA \\
\hline Riqueza observada $(S)$ & 5 & 9 & 8 & 4 & 12 & 9 & 10 & 15 \\
Abundância amostrada $(M)$ & 10 & 19 & 11 & 17 & 26 & 27 & 25 & 36 \\
Diversidade de Shannon $(H)$ & 1,25 & 1,90 & 2,02 & 0,79 & 2,26 & 1,53 & 1,78 & 2,19 \\
Dominância de Simpson [ $\left.D_{(1 / D)}\right]$ & 3,45 & 6,56 & 7,12 & 1,65 & 9,66 & 3,05 & 3,74 & 11,89 \\
Equitabilidade de Pielou(J) & 0,78 & 0,87 & 0,97 & 0,57 & 0,91 & 0,70 & 0,81 & 0,81 \\
\hline Legenda: Sil - Praça Dr. Silveira; CoG - Praça Coelho Gomes; Con - Praça da Concórdia; MJP - \\
Praça Marechal José Pessoa; Cen - Praça do Centenário; JoM - Praça Dr. João Maia; OIB - Praça \\
Dr. Oliveira Botelho; PaA - Parque do Aarão
\end{tabular}

\section{Similaridade entre as áreas de estudo}

O índice de similaridade de Jaccard demonstrou que há dissimilaridade de espécies entre as áreas amostradas, uma vez que as variações foram entre $S_{J}=0,07$ e $S_{J}=0,23$ (Praças Concórdia e Dr. João Maia), como se pode observar na Figura 2. Para MüellerDombois e Ellenberg (1974), duas ou mais áreas são consideradas similares, em termos de composição florística, quando apresentam pelo menos $25 \%$ de espécies comuns.

Provavelmente, os valores de similaridades encontrados sejam um reflexo da diferença temporal de construção de cada área e o modelo paisagístico utilizado por gestões municipais diferentes, não havendo preocupação com a consolidação de uma identidade florística urbana na paisagem da região. 
Figura 2. Dendrograma de similaridade por Jaccard entre as áreas estudadas.

Figure 2. Dendrogram of Jaccard similarity between the studied areas.

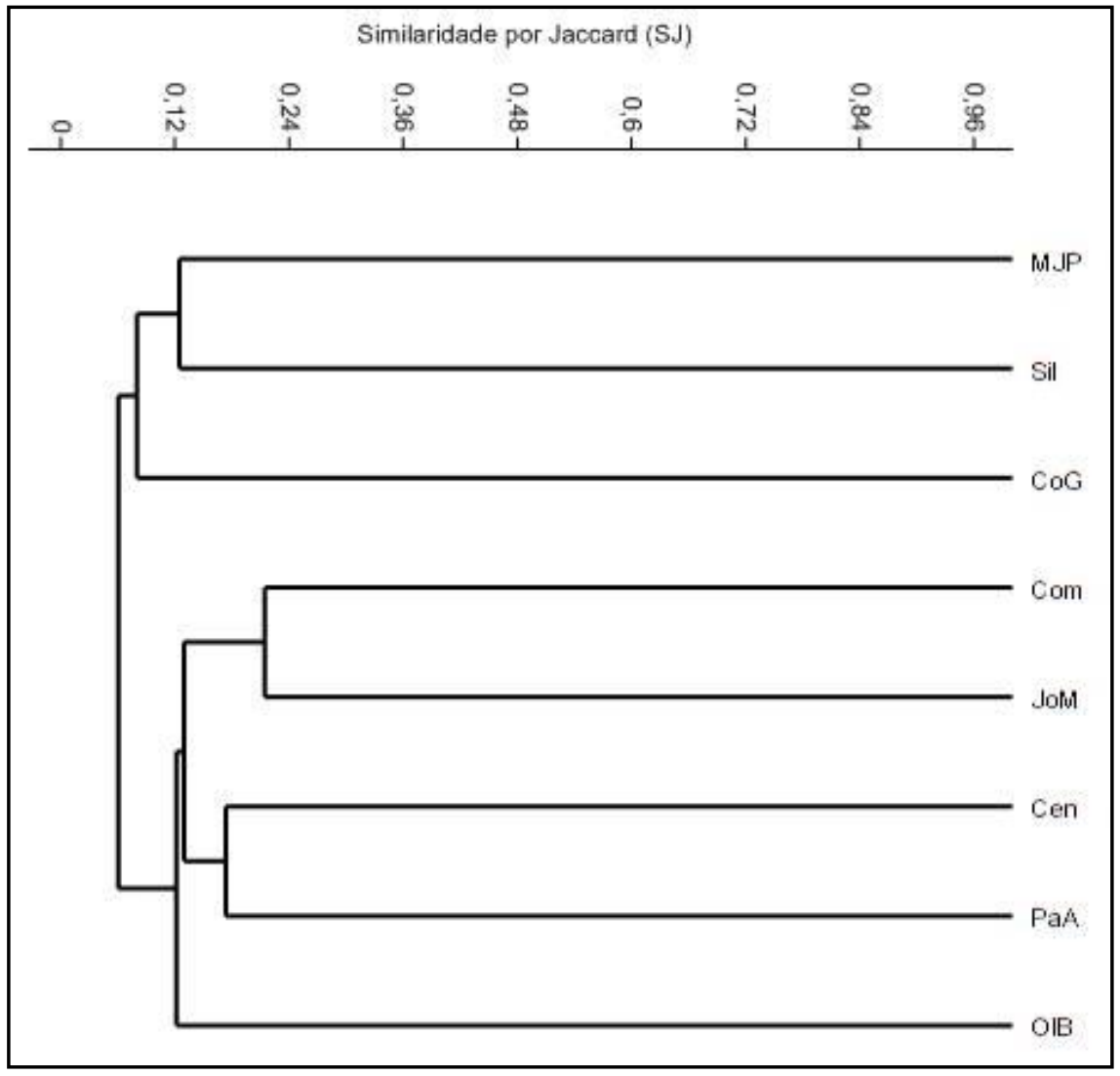

Legenda: Sil - Praça Dr. Silveira; CoG - Praça Coelho Gomes; Com - Praça da Concórdia; MJP Praça Marechal José Pessoa; Cen - Praça do Centenário; JoM - Praça Dr. João Maia; OIB - Praça Dr. Oliveira Botelho; PaA - Parque do Aarão

\section{Composição estrutural e sua relação de interferências ao patrimônio material}

Entre os espécimes amostrados, apenas 16,95\% (N=29) dos indivíduos apresentavam altura igual ou inferior a 5 metros, evidenciando assim, que o plantio foi feito com mudas bem adaptadas às condições locais ou devidamente rustificadas antes do plantio. Em relação às alturas e aos DAP's registrados, houve uma maior frequência de indivíduos entre a classe de 6 a 10 m (59,06\%; N=101) e 21 a $30 \mathrm{~cm}$ (23,39\%; N=40), respectivamente (Figuras 3 e 4), indicando uma ocorrência no predomínio de árvores adultas possivelmente inseridas em épocas diferentes no processo de construção áreas públicas. 
Interferências negativas registradas, como: comprometimento do dossel na fiação, avanço da copa na rua (Figura 5) e a destruição de calçadas (Figura 6) foram constatadas como baixa frequência. Muito embora não correlacionado estatisticamente, observações em campo sugerem que espécimes com elevada altura e DAP são as maiores responsáveis por interferências a fiação, avanço da copa sobre as ruas e destruição de calçadas por exposição do sistema radicular, sendo que os indivíduos de $F$. benjamina foram responsáveis pela maioria dos danos registrados. Em geral, espécies como $F$. benjamina necessitam de melhor planejamento público antes do seu plantio em determinadas áreas, já que os custos de manutenção e danos a patrimônios podem superar os benefícios advindos da espécie.

Com relação à disposição das árvores (Figura 7) foi observado que 62,57\% ( $n=107)$ dos indivíduos encontravam-se entrelaçados, evidenciando competição espacial nos estratos superiores das árvores (copa). Outro fator observado foi a relação entre as copas e as casas, onde $91,81 \%(n=157)$ dos indivíduos não possuíram relação direta com as edificações avaliadas, o que sugerem respeito a uma distância mínima de segurança entre as edificações e áreas estudadas pré-estabelecidas pelo código de obras municipal.

Figura 3.Distribuição de indivíduos amostrados por classes de altura (m)

Figure 3. Distribution of individuals sampled for height classes (m)

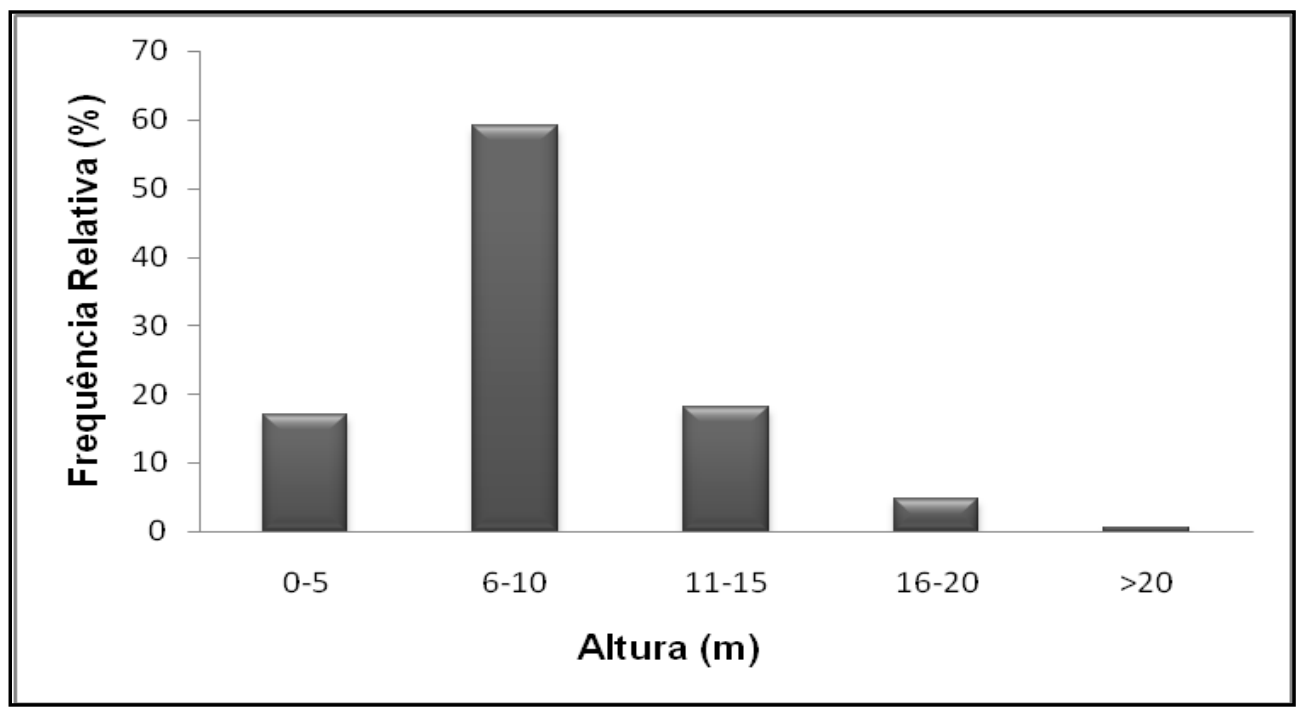


Figura 4. Distribuição de indivíduos amostrados por diâmetro da altura do peito (DAP: $\mathrm{cm}$ )

Figure 4. Distribution of individuals sampled by diameter breast height (DAP: $\mathrm{cm}$ )

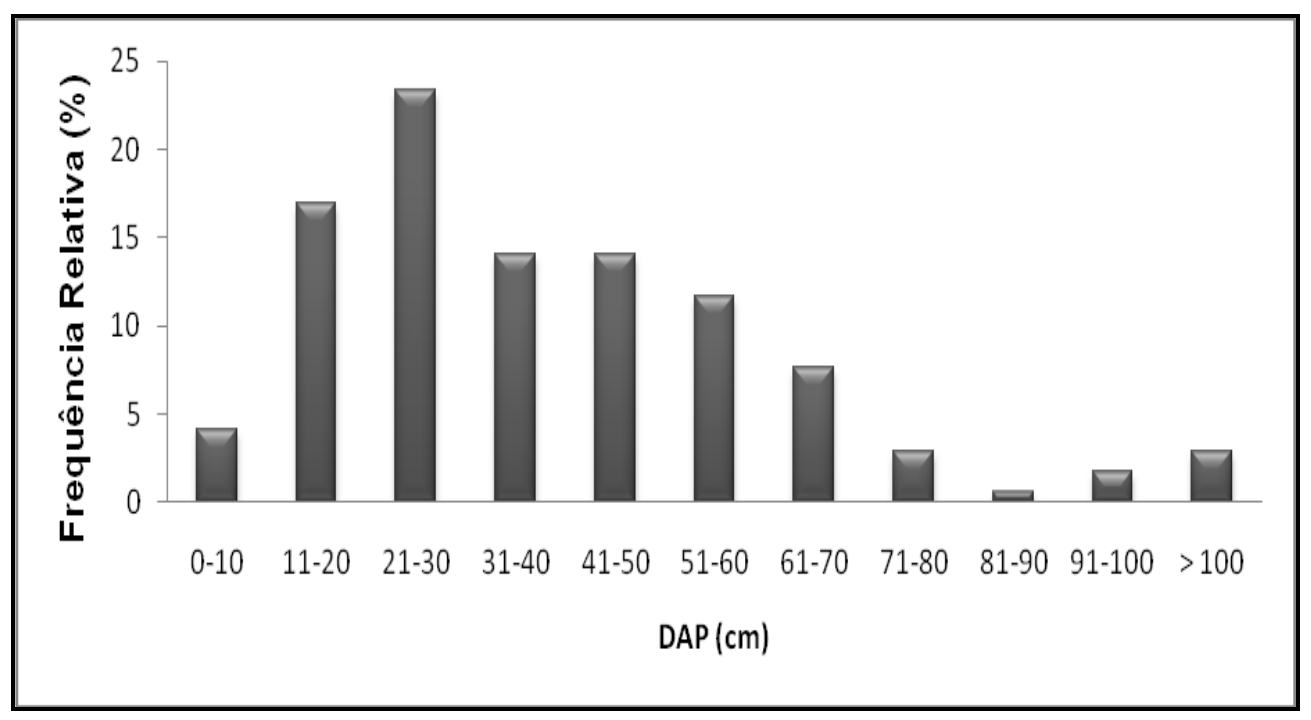

Figura 5. Distribuição de indivíduos amostrados e sua relação na interferência de patrimônios pela copa

Figure 5. Distribution of sampled individuals and their relationship in the interference of patrimonies by the canopy

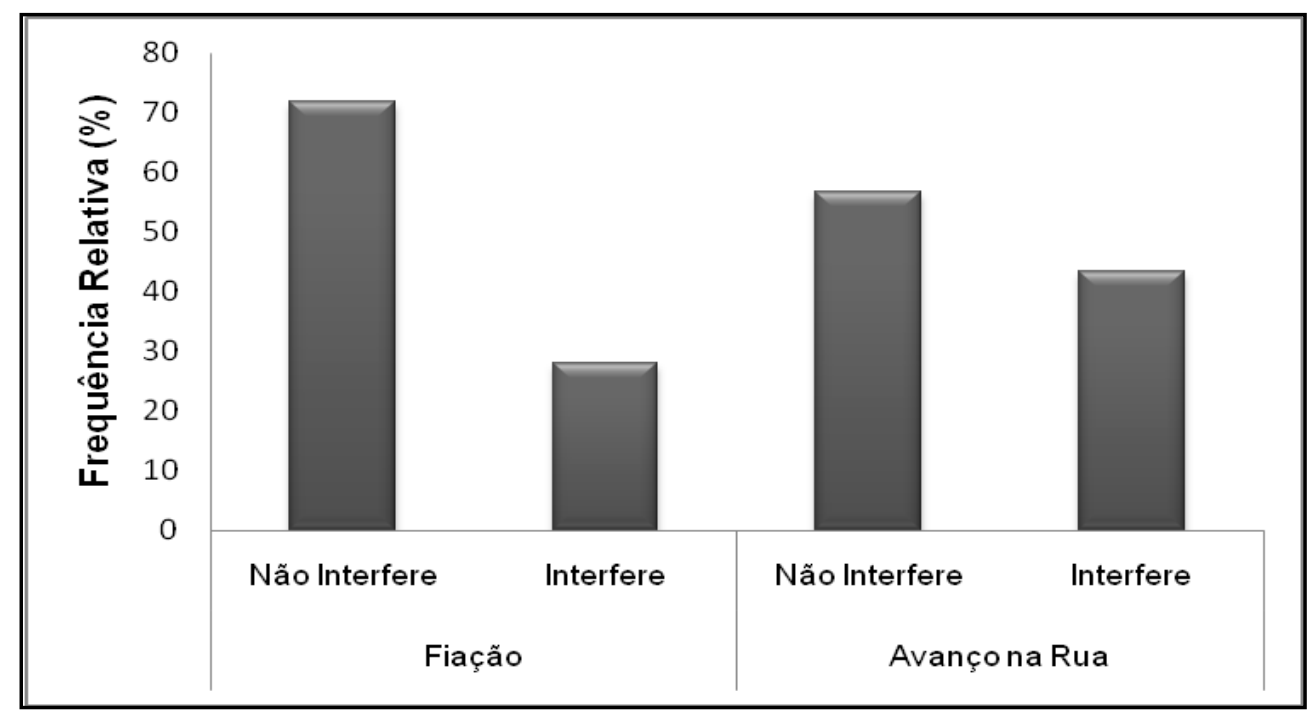


Figura 6. Distribuição de indivíduos amostrados e sua relação na interferência de patrimônios pelas raízes

Figure 6. Distribution of sampled individuals and their relationship in the interference of patrimonies by the roots

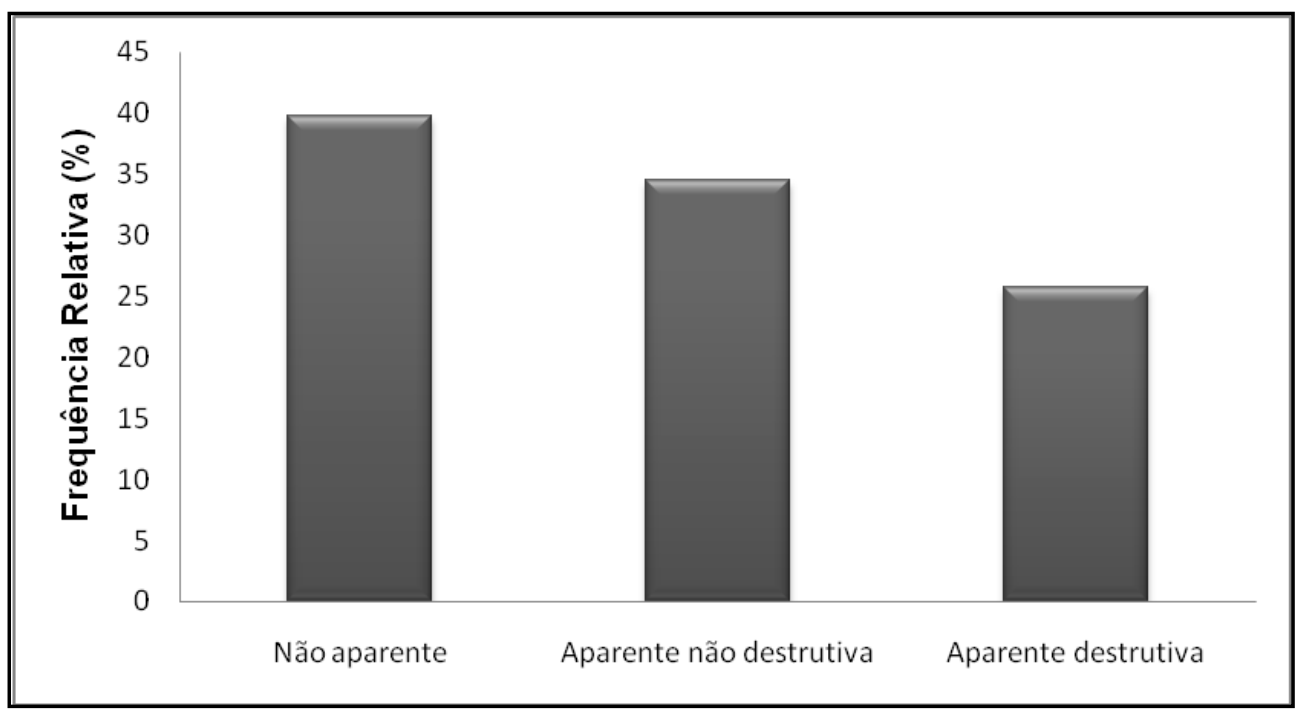

Figura 7.Distribuição de indivíduos amostrados e a forma de disposição das copas das árvores

Figure 7. Distribution of sampled individuals and the formof disposal the treetops

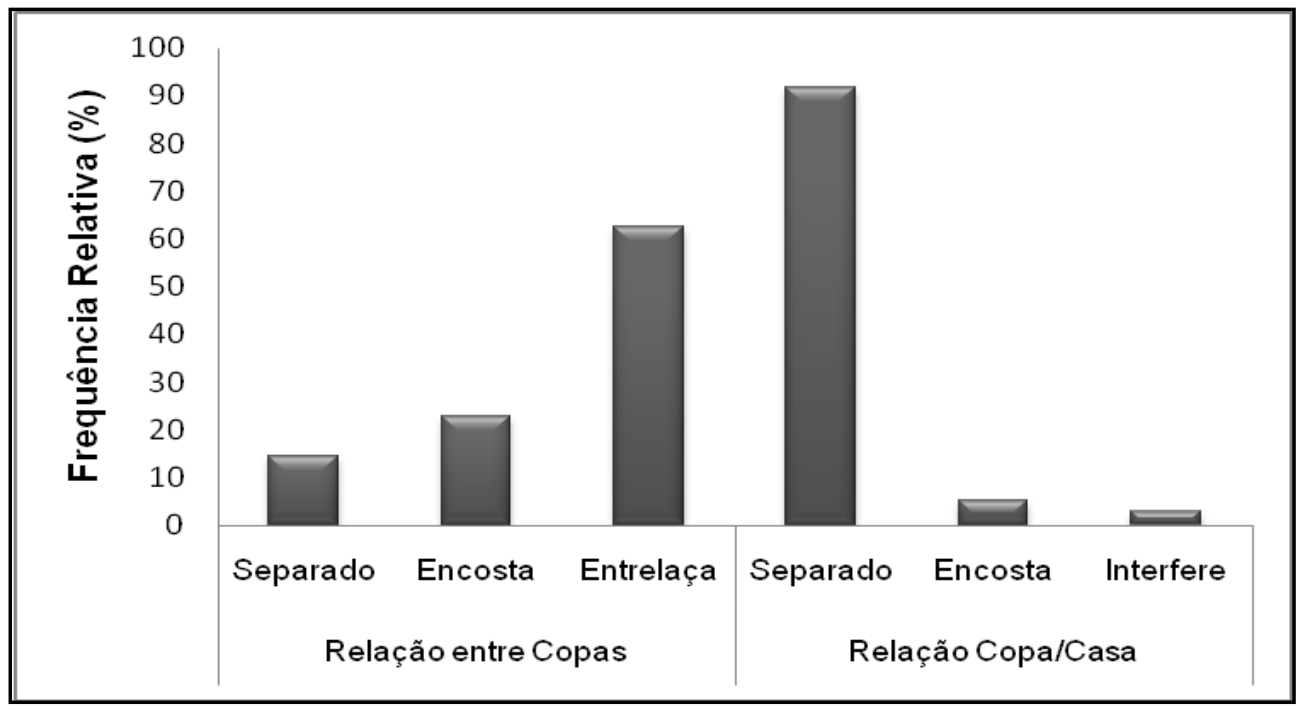

\section{Aspectos ecológicos e de fitossanidade}

Os aspectos de fitossanidade avaliados entre os indivíduos e suas respectivas áreas de plantio demonstraram que $1,16 \%(\mathrm{~N}=2)$ encontravam mortos, 12,28\% ( $\mathrm{N}=21)$ foram 
considerados maltratados devido à abundância elevada de espécies de plantas parasitas em galhos e evidência de muitos danos no tronco arbóreo (cortes, presença de pregos para disposição de gaiolas de pássaros, pichação, entre outros). Quarenta e três por cento $(\mathrm{N}=74)$ foram considerados regulares, uma vez que possuíam algum tipo de interferência negativa registrado, porém sem maiores comprometimentos ao bem-estar dos espécimes, enquanto os demais foram considerados com boa vitalidade $(43,27 \%$; $N=74$ : ausência de parasitas e outras interferências negativas). Brianezi et al. (2013), Sousa et al. (2013) e Sousa et al. (2014), afirmaram que a predominância de espécies com boa vitalidade, mesmo sem grande cuidado pelo poder público ou pela população, reflete boa adaptados às condições urbanas, tanto climáticas quanto antrópicas.

No total de $55 \%(n=94)$ das árvores amostradas apresentavam algum sinal de injúria ou agressão mecânica, sendo mais ocorrente nas áreas com uma maior movimentação da comunidade, corroborando, em parte, com a afirmação de Matos et al. (2010) de que as árvores que emolduram as praças urbanas são vítimas eminentes nos períodos festivos, tendo em vista que comumente são utilizadas como suporte para ornamentação, o que demanda o uso de instrumentos de fixação dos adornos, como pregos e grampos.

As análises de caracterização no manejo de dossel descreveram que em 23,97\% $(\mathrm{n}=41)$ dos indivíduos não existiu a necessidade de nenhum tipo de manejo (limpeza ou poda); $36,25 \%$ ( $n=32$ ) necessitavam de uma poda leve com a retirada de alguns galhos; $31,57 \%$ ( $n=54)$ necessitavam de uma poda pesada com a remoção de grande quantidade dos galhos, enquanto $8,18 \%(n=14)$ precisam de poda drástica, por estarem mortos ou com muitos danos, podendo ocasionar futuros problemas a edificações ou pessoas (Figura 8).

Figura 8. Distribuição de indivíduos amostrados quanto à necessidade de manejo no dossel Figure 8. Distribution of sampled individuals as the need to manage the canopy

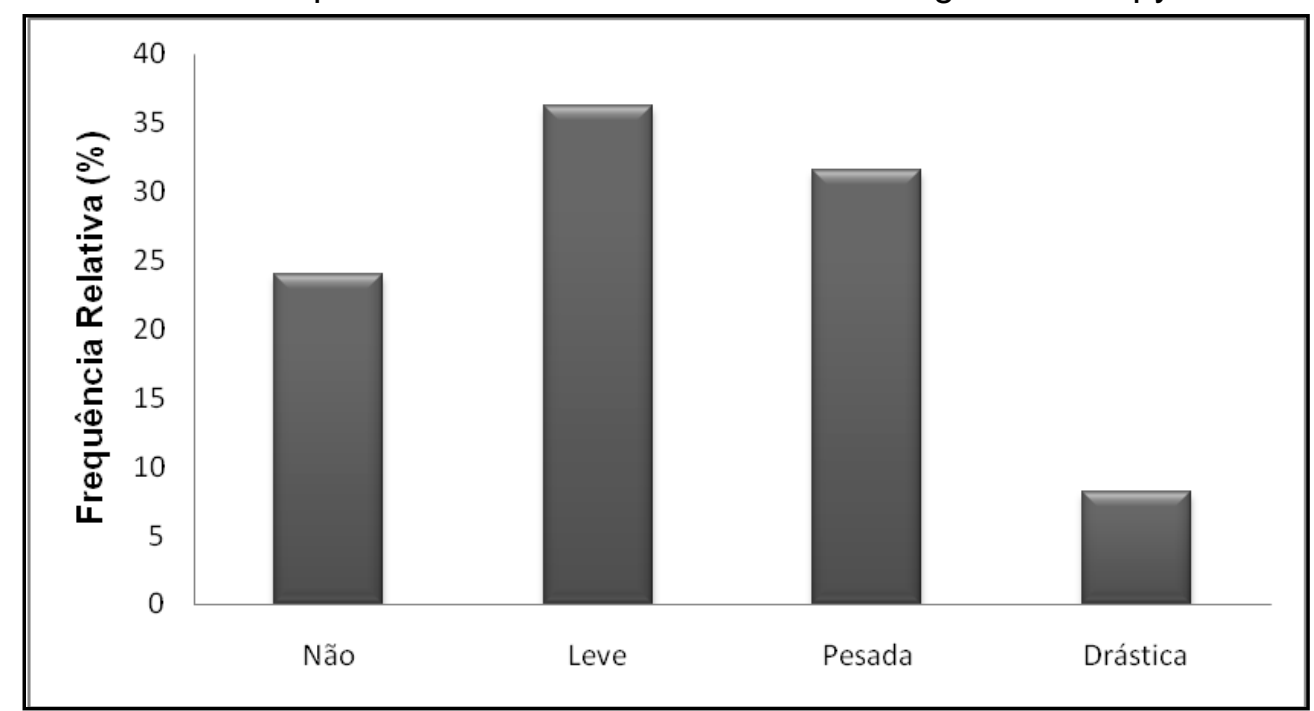


Segundo Seitz (1990) na execução de podas, deve ser dada atenção especial à morfologia da base do galho, pois na inserção do galho no tronco principal, distinguem-se duas estruturas: a crista na parte superior e o colar na parte inferior da base do galho, que são de vital importância para garantir a cicatrização da planta. Quando ocorre uma poda mal executada, o indivíduo pode ser infectado através desta lesão, podendo levá-lo a morte.

Outro aspecto observado em várias ocasiões é a deformação das árvores após a tentativa de poda drástica. Nesses casos ocorre a rebrota de galhos próximo ao local de poda, assim, o indivíduo volta a se desenvolver, porém sem a sua orientação original.

Em relação às interações biológicas, foram observados que $77,77 \%(n=133)$ dos indivíduos possuíam relações com alguma espécie de epífita (ex. Tillandsia sp., Microgramma sp.) (Figura 9). Em contrapartida, foram registrados em 11,11\% $(n=19)$ dos indivíduos amostrados presença de plantas parasitas, como a popularmente conhecida erva-de-passarinho.

Figura 9. Distribuição de indivíduos amostrados quanto ao tipo de interação ecológica

Figure 9. Distribution of sampled individuals as the type of ecological interaction

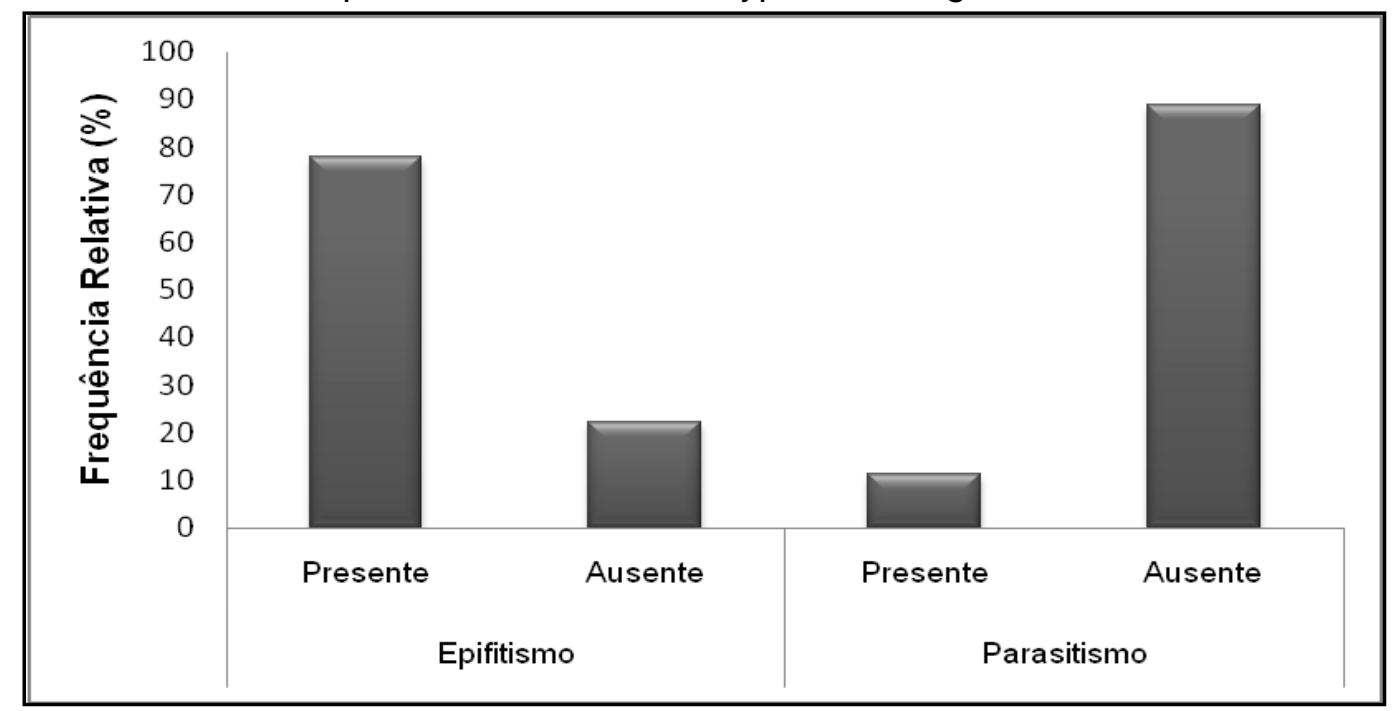

Para Rotta et al. (2005), a erva-de-passarinho é comumente confundida com algumas epífitas que se desenvolvem nos troncos e galhos das árvores urbanas, sendo as mais comuns Microgramma squanulosa (Polypodiaceae) e Tillandsiastricta (Bromeliaceae).

Estas plantas não ocasionam danos diretos ao hospedeiro (retirada dos elementos minerais), sendo fácil e sem problemas a sua retirada ou controle. Entretanto, dependendo da intensidade de recobrimento, podem interferir no processo de respiração através da casca, afetando sua vitalidade e, também, oportunizando condições para ocorrência de 
danos causados por insetos que habitam este ambiente (perfuração da casca e possibilidade de contaminação por agentes patogênicos).

\section{CONCLUSÕES}

As áreas públicas apresentaram elevada riqueza de espécies e bom estado fitossanitário dos indivíduos analisados. A dissimilaridade encontrada entre as áreas verdes (praças e parque) já era esperada, já que entende-se que a gestão pública, os modelos de paisagismo adotados e as influência da cultura local podem variar largamente no tempo (história do município de Resende), principalmente, em cidades que não apresentam um planejamento voltado ao manejo silvicultural urbano à longo prazo.

Recomenda-se o uso de placas informativas (identificação nas árvores) e o incentivo no planejamento de atividades práticas com estudantes poderia fomentar a criação de uma identidade na população do município com as espécies nativas plantadas e consolidar o pensamento da conservação de espécies.

\section{AGRADECIMENTOS}

Ao arquiteto Lucas Nunes pela grande ajuda na medição das áreas e pelas imagens cedidas. Aos funcionários da AGEVAP pelo apoio no desenvolvimento do estudo e aos referees pelas críticas e sugestões ao paper.

\section{REFERÊNCIAS}

APG III. An update of the Angiosperm Phylogeny Group Classification for the Orders and Families of Flowering Plants: APG III. Botanical Journal of the Linnean Society 161:105121. 2009.

BRIANEZI, D.; JACOVINE, L. A. G.; Gonçalves,W.; Rocha, S. J. S. S. Avaliação da Arborização no Campus-Sede da Universidade Federal de Viçosa. Revista da Sociedade Brasileira de Arborização Urbana, Piracicaba - SP, v.8, n.4, p 89-106, 2013.

BRUMMITT, R. K.; POWEL, C. E. Authors of Plant Names. Royal Botanic Gardens, Kew, Great Britain. 732 p. 1992. 
COSTA, L. A. C.; HIGUCHI, N. Arborização de Ruas de Manaus: Avaliação Qualitativa e Quantitativa. Revista Árvore, Viçosa, v. 23, n. 2, p. 223-232, 1999.

DANTAS, C. I.; DE SOUZA, C. M. C. Arborização urbana na cidade de Campina Grande -PB: Inventário e suas espécies. Revista de biologia e ciências da terra, Campina Grande, v. 4, n. 2, 2004.

FERREIRA, F. A.; GASPAROTTO, L.; LIMA, M. I. P. M. Uma ferrugem, causada por Phakopsoratomentosae sp. nov.,em oiti, em Manaus-AM. Fitopatologia brasileira, Brasília - DF, v.26, n.2, 2001.

HAMMER, O.; HARPER, D. A. T.; RIAN, P. D. 1999-2011. Past: Paleontological Statistics Software Package for Education and Data Analysis. Disponível em<http://folk.uio.no/ ohammer/past/index.html>. Acesso em: 11 de novembro de 2013.

INSTITUTO BRASILEIRO DE GEOGRAFIA E ESTATÍSTICA - IBGE 2010a. Sinopse do Censo Demográfico 2010. Disponível em <http://www.censo2010.ibge.gov.br/ sinopse/index. .php?dados=29\&uf=33>. Acesso em: 04 janeiro de 2013 .

INSTITUTO BRASILEIRO DE GEOGRAFIA E ESTATÍSTICA - IBGE 2010b. Manual do Recenseador, Pesquisa de Avaliação da Cobertura da Coleta 2010. Disponível em $<$ http://biblioteca.ibge.gov.br/visualizacao/instrumentos_de_coleta/doc2374.pdf>. Acesso em: 08 fevereiro de 2013.

LINDENMAIER, D. S.; SANTOS, N. O. Arborização Urbana das Praças de Cachoeira do Sul-RS-Brasil: Fitogeografia, Diversidade e Índice de Áreas Verdes. Pesquisas, Botânica, N 59: 307-320. São Leopoldo: Instituto Anchietano de Pesquisas, 2008.

LINDENMAIER, D. S.; SOUZA, B. S. P. Arborização Viária de Cachoeira do Sul/RS: Diversidade, Fitogeografia e Conflitos com a Infraestrutura Urbana. Revista da Sociedade Brasileira de Arborização Urbana, Piracicaba - SP, v.9, n.1, p 108-122, 2014.

LIRA, R. S.; DANTAS, I. C.; CAVALCANTI, M. L. F.; BARROS, M. J. B.; LIRA, V. M.; CARNEIRO, P. T. Diagnóstico Paisagístico do Parque da Criança em Campina Grande, PB. Revista de Biologia e Ciências da Terra, Sergipe - PB, v.4. n.1, p. 1-23, 2004.

LORENZI, H. Árvores Brasileiras: Manual de Identificação e Cultivo de Plantas Arbóreas do Brasil. vol. I. Ed. 1. Nova Odessa, SP: Instituto Plantarum, 2002a.

LORENZI, H. Árvores Brasileiras: Manual de Identificação e Cultivo de Plantas Arbóreas do Brasil, vol. II. Ed. 2. Nova Odessa, SP: InstitutoPlantarum, 2002b.

MAGURRAN, A. E. Measuring Biological Diversity. Blackwell Science, Oxford. 2004. 
MATOS, E. C. A.; NASCIMENTO-JÚNIOR, J. E.; MARIANO, D. L. S.; OLIVEIRA, A. L. Arborização do bairro Centro da Cidade de Aracaju, Sergipe, e seus Organismos Associados. Rev. da Sociedade Brasileira de Arborização Urbana, Piracicaba, v. 5, n. 4, p. 22-39, dez. 2010.

MELO, R. R.; LIRA FILHO, J. A.; RODOLFO JÚNIOR, F. Diagnóstico Qualitativo e Quantitativo da Arborização Urbana no Bairro Bivar Olinto, Patos, Paraíba. Revista da Sociedade Brasileira de Arborização Urbana, Piracicaba, v. 2, n. 1, 2007.

MIRANDA, T. O.; CARVALHO, S. M. Levantamento Quantitativo e Qualitativo de Indivíduos Arbóreos Presentes nas Vias do Bairro da Ronda em Ponta Grossa-PR. Revista da Sociedade Brasileira de Arborização Urbana, Piracicaba - SP, v.4, n.3, p.143 - 157, 2009.

MUELLER-DOMBOIS, D.; ELLENBERG, H. Aims and Methods Vegetation Ecology. New York: J. Wiley . 1974.

PAIVA, G. A. Levantamento Florístico Quali-Quantitativo da Praça do Relógio da Cidade Universitária "Armando de Salles Oliveira" - USP - Piracicaba/SP. 116 p. Dissertação (Mestrado em Agronomia). Escola Superior de Agricultura Luiz de Queiroz USP, 2006.

PREFEITURA MUNICIPAL DE RESENDE - PMR. Informações Geofísicas. Disponível em <http://www.resende.rj.gov.br/info_geofisicas.asp>. Acesso em: 04 janeiro de 2013.

ROMANI, G. N.; GIMENES, R.; SILVA, M. T.; PIVETTA, K. F. L.; BATISTA, G. S. Análise Quali-Quantitativa da Arborização na Praça XV de Novembro em Ribeirão Preto - SP, Brasil. Revista Árvore, Viçosa-MG, v.36, n.3, p. 479-487, 2012.

ROSSATTO D. R.; TSUBOY, M. S. F.; FREI, F. Arborização urbana na cidade de Assis-SP: uma abordagem quantitativa. Revista da Sociedade Brasileira de Arborização Urbana, Piracicaba - SP, v. 3, n. 3, p. 1-16, 2008.

ROTTA, E.; OLIVEIRA, Y. M. M.; ARAÚJO, A. J., INOUE, M. T. Reconhecimento Prático de Cinco Espécies de Erva-de-Passarnho na Arborização de Curitiba, PR. Ed. 21. Colombo: Embrapa Florestas, 2005.

SANCHOTENE, M. C. C. Desenvolvimento e Perspectivas da Arborização Urbana no Brasil. In: CONGRESSO BRASILEIRO DE ARBORIZAÇÃO URBANA, 1994, São Luis. Anais,.. São Luís, 1994, p. 15-25.

SANTAMOUR JÚNIOR, F. S. Trees for Urban Planting: Diversity Uniformity, and Common Sense. In: METRIA CONFERENCE 7. 1990, Lisle. Anais... Proceedings. Lisle: p. 57-66. 1990. 
SEITZ, R. A. Considerações sobre a poda de árvores na arborização urbana. In: ENCONTRO NACIONAL SOBRE ARBORIZAÇÃO URBANA, 3, Curitiba-PR, 1990. Anais... Curitiba: Fupef. 1990 p. 87-100.

SILVA JÚNIOR, O. A. B.; MÔNICO, M. O. M. Arborização em Harmonia com a Infraestrutura Urbana. In: $1^{\text {a }}$ Semana de Meio Ambiente. Prefeitura Municipal de Guarulhos: Secretaria de Meio Ambiente, 1994.

SILVA, G. C. Questões Ambientais, Culturais e Socioeconômicas de Espaços Livres Urbanos: Praças do Centro da Cidade de Teresina-PI. 172f. Dissertação (Mestrado em Desenvolvimento e Meio Ambiente). Universidade Federal do Piauí, Teresina. 2009.

SOUSA, L. M.; FIGUEIRÊDO, M. F.; BRAGA, P. E. T. Levantamento Quali-Quantitativo da Arborização Urbana do Distrito de Rafael Arruda, Sobral, CE. Revista da Sociedade Brasileira de Arborização Urbana, Piracicaba - SP, v.8, n.3, p 118-129, 2013.

SOUSA, R. C.; AGUIAR, O. T.; SILVA, L. T. A.; SILVA, L. A.; MARRA, R. C. Avaliação Quali-Quantitativa da Arborização na Praça Agostinho Nohama, Bairro Lauzane Paulista, São Paulo - SP. Revista da Sociedade Brasileira de Arborização Urbana, Piracicaba SP, v.9, n.1, p 92-107, 2014.

TEIXEIRA, I. F. Análise qualitativa da arborização de ruas do conjunto habitacional Tancredo Neves, Santa Maria - RS. Ciência Florestal, Santa Maria - RS, V.9, n.2. p. 9-21, 1999.

ZILLER, S. R. Os processos de Degradação Ambiental Originados por Plantas Invasoras. Revista Ciência Hoje, São Paulo - SP, n.178, p. 77-79, dez. 2001. 